\title{
Observation of the Pitch Angle Distribution of High-Energy Ions and their Radial Transport in the Central Cell of GAMMA10*)
}

\author{
Yasutaka IMAI, Makoto ICHIMURA, Ryuya IKEZOE, Tatsuya MURAKAMI, Toru IWAI, \\ Takuro YOKOYAMA, Yumeto UGAJIN, Tatsunori SATO and Tsuyoshi IMAI \\ Plasma Research Center, University of Tsukuba, Tsukuba 305-8577, Japan
}

(Received 7 December 2010 / Accepted 8 March 2011)

\begin{abstract}
Suppression of the radial transport of the particles caused by low-frequency density fluctuations is one of the important subjects in the GAMMA10 tandem mirror. In GAMMA10, plasmas are mainly produced and heated with Ion Cyclotron Range of Frequency (ICRF) waves. The plasmas heated with ICRF waves have strong temperature anisotropy, because the ions are accelerated perpendicularly to the magnetic field line. In the GAMMA10 experiments, two types of low-frequency density fluctuations are observed; one is a drift-type fluctuation and the other is a flute-type fluctuation. In this paper, we study the radial transport of ICRF-produced high-energy ions which is caused by the low-frequency density fluctuations.
\end{abstract}

(c) 2011 The Japan Society of Plasma Science and Nuclear Fusion Research

Keywords: tandem mirror, drift-type fluctuation, flute-type fluctuation, pitch angle distribution, radial transport

DOI: $10.1585 /$ pfr.6.2402152

\section{Introduction}

Stable plasmas with ion temperature of several $\mathrm{keV}$

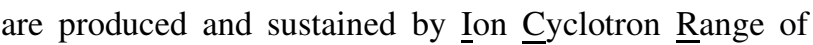
Frequency (ICRF) waves in GAMMA10. The plasmas heated near the central cell midplane with ICRF waves have strong temperature anisotropy, because ions are accelerated perpendicularly to the magnetic field line.Lowfrequency fluctuations are observed in relation to applied ICRF powers and the amount of injecting gas. Two types of low-frequency fluctuations are observed; one is a drifttype fluctuation and the other is a flute-type fluctuation. The drift-type fluctuations are observed in the case of highpower ICRF experiments. The flute-type fluctuations are observed in the case of electron cyclotron heating $(\mathrm{ECH})$ experiments. Due to the potential formation in plug/barrier cells, centrifugal force of the $E \times B$ rotation will drive the flute-type instability. Interactions of ions with such lowfrequency fluctuations can be one of possible candidates for the particle transport in the radial direction across the magnetic field line. In this paper, the radial transport of high energy ions caused by two types of density fluctuation is investigated experimentally.

\section{GAMMA10 Device}

GAMMA10 consists of five mirror cells which are a central cell, two minimum- $B$ anchor cells placed outside the central cell, and the plug/barrier cells at both ends. The central cell has an axisymmetric mirror configuration and is $5.6 \mathrm{~m}$ in length with the magnetic field

\footnotetext{
author'se-mail: imai_yasutaka@prc.tsukuba.ac.jp

*) This article is based on the presentation at the 20th International Toki Conference (ITC20).
}

strength of $0.4 \mathrm{~T}$ at the midplane. The anchor cells are the minimum- $B$ configuration and are connected to both ends of the central cell. The role of anchor cells is to maintain the macroscopic stability of the GAMMA10 plasma. The plug/barrier cells have an axisymmetric configuration and are used for the formation of the electrostatic confinement potentials with ECH. Figure 1 shows the schematic drawing of GAMMA10 and axial profile of magnetic field strength. Two ICRF sources (RF1 and RF2) are used for plasma production, heating and stabilizing against the Magneto-Hydro-Dynamic (MHD) modes. For two purposes, ECH is used in GAMMA10. One is the formation of the electrostatic confinement potential in the plug/barrier region. The other is the electron heating in the central cell. The ions are confined axially by the magnetic mirror effect and the positive plug potential.

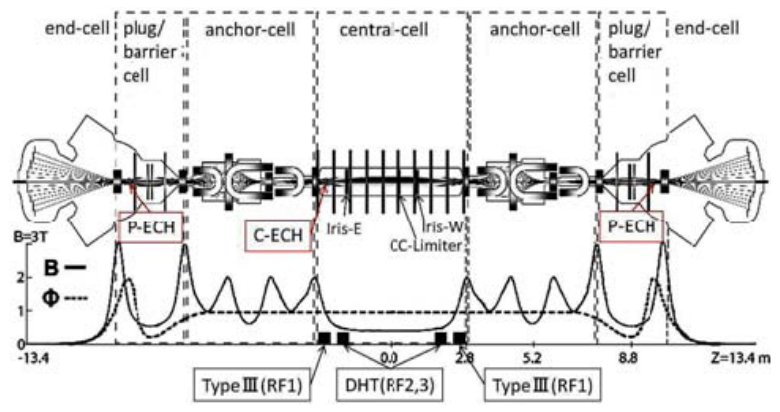

Fig. 1 Schematic drawing of GAMMA10 and axial profile of magnetic field strength. 


\section{Diagnostics}

\subsection{Electrostatic probes for measuring fluc- tuations}

Density fluctuations and their mode structures are measured by electrostatic probes (ESP) arrayed in the azimuthal and axial directions on the peripheral region of the central cell. Drift-type fluctuations propagate in the direction of the electron diamagnetic motion and also in the axial direction.Flute-type fluctuations are detected as propagating waves in the ion diamagnetic direction due to the $E \times B$ rotation. It is confirmed that the flute-type fluctuation has no wave numbers in the axial direction.

\subsection{Central-cell high-energy ion detector (ccHED) for measuring behavior of high energy ions}

To measure the behavior of the high energy ions, central-cell High Energy-ion Detector (ccHED) is installed in the central cell. A silicon surface barrier (SSB) diode is used for the detector. ccHED is located in the peripheral region just at the midplane of the central cell and can detect high-energy ions which escape from the core region to the perpendicular direction. Figure 2 shows the schematic drawing of ccHED. ccHED has a pin hole of which diameter is $0.5 \mathrm{~mm}$ exists in front of the SSB detector on the inner

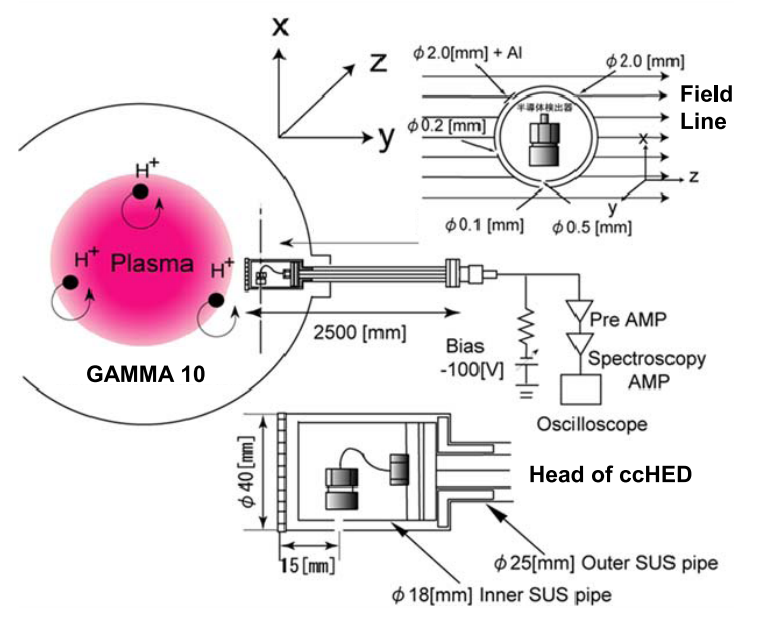

Fig. 2 Schematic drawing of ccHED.

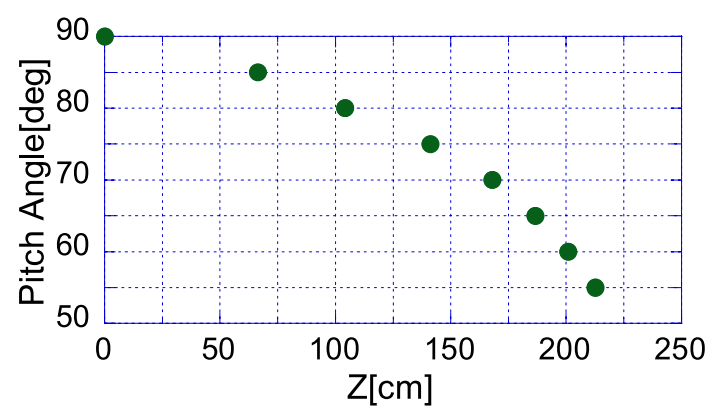

Fig. 3 Relation between pitch angle and the location of the reflection point, $Z$. pipe. On the outer pipe, pin holes with various diameters are set upon the surface. ccHED can measure high energy ions of which energy is above $10 \mathrm{keV}$ [1]. The Larmor radius of $10 \mathrm{keV}$ proton is about $2.5 \mathrm{~cm}$ and is larger than the size from top of ccHED to the pin hole. By rotating both pipes against to the magnetic field line, a pitch angle distribution of the ions can be measured. The total number of high energy ions and resolution of the pitch angle are changed by using the pin hole with a different diameter on the outer pipe. When a size aperture of which diameter is $0.2 \mathrm{~mm}$ is used, the pitch angle resolution becomes \pm 3.3 degrees. Pitch angles of high energy ions measured at the midplane correspond to turning points of mirror confined ions in the central cell.

Frequency of RF2 of $6.36 \mathrm{MHz}$ has resonance layers at $z= \pm 117 \mathrm{~cm}$. The ions which pass through these layers are accelerated perpendicularly to the magnetic field line. Accelerated ions have large pitch angle. The location of the cyclotron resonance layer corresponds to the pitch angle of 80 degrees at the midplane. High energy ions are detected mainly near the pitch angle of 80 degree. Figure 3 shows the pitch angles of ions which are reflected at the axial location of $Z$.

\section{Experimental Result}

\subsection{Interaction with drift-type fluctuations}

Figure 4 shows the temporal evolution of frequency spectrum of (a) ion saturation current measured by ESP and (b) signal of high energy ions by ccHED. As shown in Fig. 4 (a), drift-type fluctuations of which frequencies are near $5 \mathrm{kHz}$ are observed strongly from $100 \mathrm{~ms}$ to $150 \mathrm{~ms}$. In the azimuthal direction, these density fluctuations have a mode number of $m=+1$, which means a rotation in
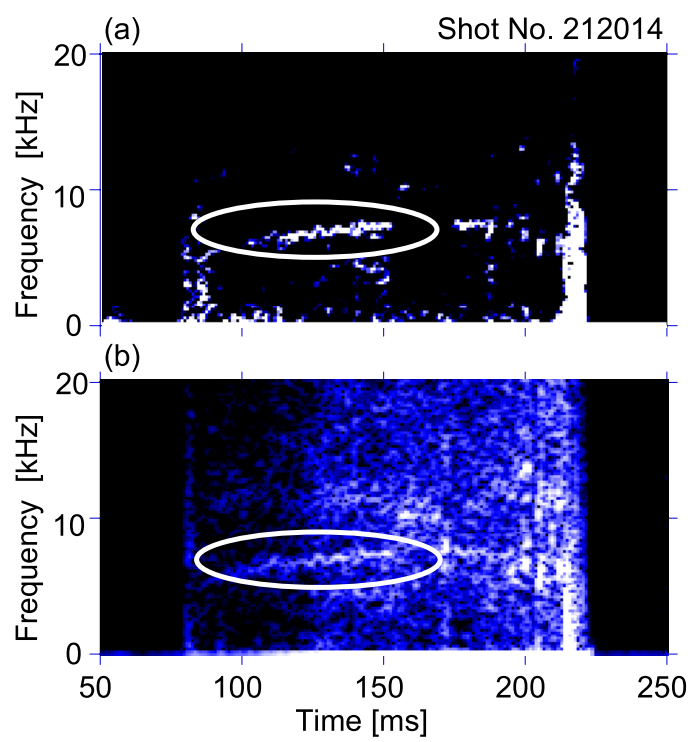

Fig. 4 Intensity plots of frequency spectra of (a) ESP and (b) ccHED. 


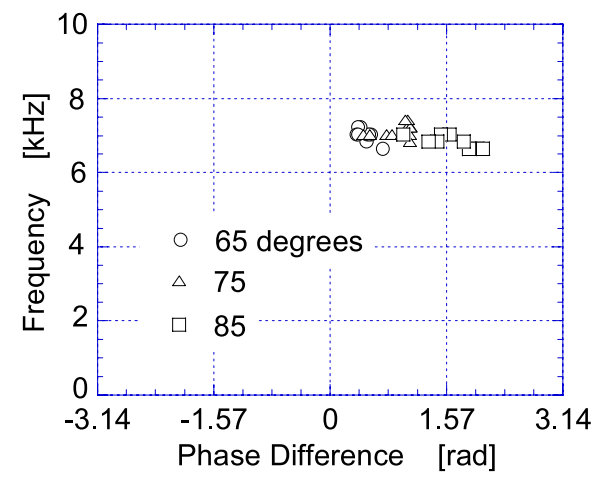

Fig. 5 Phase differences in cases of 65, 75 and 85 degrees.

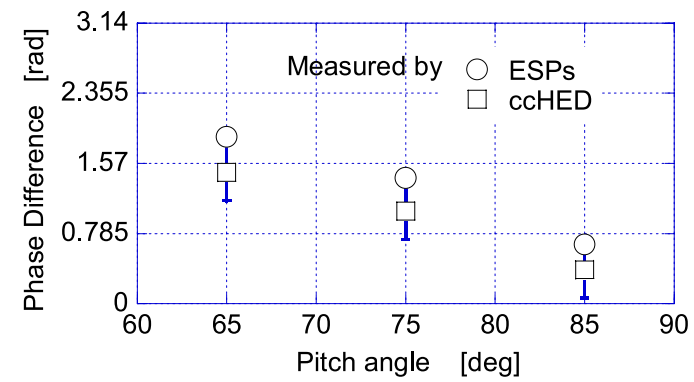

Fig. 6 Relation between phase difference and pitch angle.

the electron diamagnetic direction. As shown in Fig. 4 (b), the spectrum is rather noisy compared to that of the ESP signal, however, a clear peak with the same frequency as that in the ESP signal is identified [2]. The reduction of diamagnetism is observed, when the amplitude of density fluctuations increases. In such cases, it is clearly observed that the signals of ccHED increase. This experimental result suggests that the radial transport of high energy ions is caused by the drift-type fluctuations.

The phase difference between both frequency peaks in the ESP and ccHED signals are analyzed to study the interaction of high-energy ions with the density fluctuations. Figure 5 shows detected phase differences in cases of 85 , 75 and 65 degrees. As shown in the figure, the phase differences depend clearly on the pitch angles. To consider the absolute phase difference, we assume the phase difference is zero when the pitch angle is 90 degrees because ccHED is located at the midplane $(z=0)$ in the central cell. The distance between reflection points of ions with pitch angles of 65 and 85 degrees is about $1.2 \mathrm{~m}$, which is estimated from the magnetic field profile as indicated in Fig. 3.

Figure 6 shows the pitch angle distributions of phase difference which measured by two ESPs and ccHED. From two ESPs arrayed in the axial direction, the axial wave number of the density fluctuation is estimated to be about $1.0 \mathrm{~m}^{-1}$ in this experiment. The phase difference of the density fluctuation in cases of ions with pitch angles 65 and 85 degrees becomes 1.1 radians. This is similar value as the phase difference obtained from the ccHED signals.
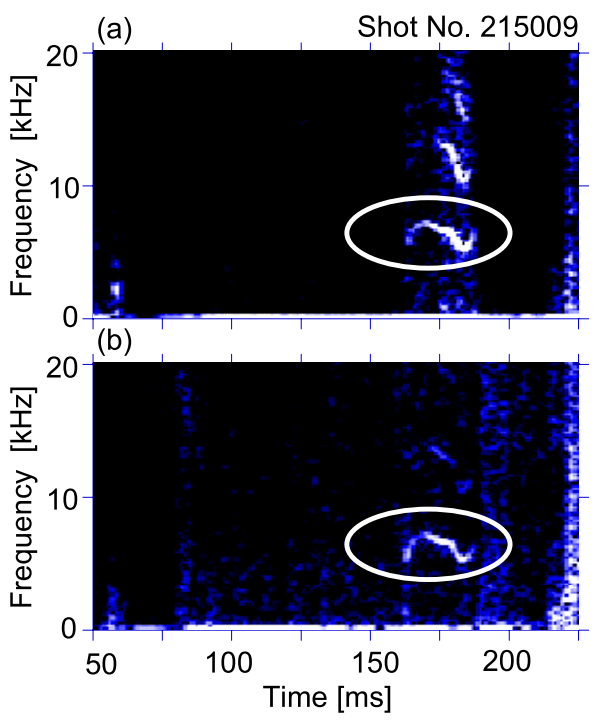

Fig. 7 Intensity plots of frequency spectra of (a) ESP and (b) ccHED.

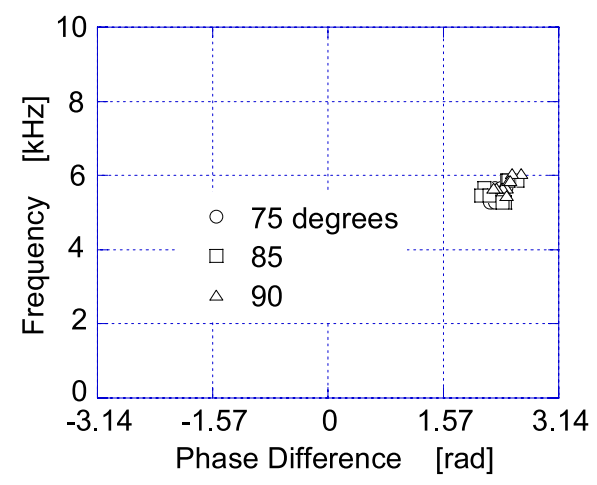

Fig. 8 Phase differences in cases of 75 degrees, 80 degrees and 90 degrees.

It will be possible to consider that ions interact with the drift-type fluctuations near the reflection points.

\subsection{Interaction with flute-type fluctuations}

Figure 7 shows the temporal evolution of frequency spectra of (a) ion saturation current measured by ESP and (b) signals of high energy ions by ccHED. As shown in figures, a flute-type fluctuation of which frequency is near $5 \mathrm{kHz}$ is observed strongly in both signals from $170 \mathrm{~ms}$ to $190 \mathrm{~ms}$. When ECH is applied from 150 to $190 \mathrm{~ms}$, a flute-type fluctuation is sometimes excited strongly. Flutetype fluctuations observed in GAMMA10 do not have finite wave number in the axial direction. To consider the pitch angle dependence of high-energy ions, we measured the pitch angle distribution in same way as drift-type fluctuations. Figure 8 shows the phase differences in the case of the same plasma sequence as shown in Fig. 7. This experimental result shows no pitch angle dependences. 


\section{Summary}

The phase differences between ccHED signals and ESP signals are measured when the drift-type fluctuations appear. The pitch angle and the phase dependence indicated that ions receive strong interaction from the density fluctuations near the reflection point and are transported in the radial direction. When the flute-type fluctuations appear, no pitch angle dependences are observed. The strong interaction near the reflection points is also suggested because the flute-type fluctuations have no wave number in the axial direction.

\section{Acknowledgements}

This study is supported by the bi-directional collaborative research program of National Institute for Fusion Science, Japan (NIFS09KUGM040). The authors acknowledge the GAMMA10 groups at the University of Tsukuba for their cooperation in the experiments.

[1] M. Ichimura et al., Rev. Sci. Instrum. 70, 834 (1999)

[2] M. Katano et al., Trans. of Fusion Sci. and Technol. 51, no.2T, 289 (2007). 